\title{
An Improved Allan Variance Method Used for the Characteristics Analysis of the Band-Pass Mixed Noise
}

\author{
Mingming Liu ${ }^{1,}$, Manman Huang ${ }^{2, b}$ \\ ${ }^{1}$ School of Electronic Information and Communications, Huazhong University of Science and \\ Technology,Wuhan, Luoyu Road, 1037, China \\ ${ }^{2}$ School of Electronic Information and Communications, Huazhong University of Science and \\ Technology,Wuhan, Luoyu Road, 1037, China \\ aemail: liu_mming@126.com, bemail: ywhsmm152@163.com
}

Keywords: Improved Allan variance method, characteristics analysis, band-pass mixed noise

\begin{abstract}
An improved Allan variance method (IAVM) is proposed for the characteristics analysis of the band-pass mixed noise to distinguish the different types of the noise in the band-pass mixed noise and give the corresponding intensity coefficients of the power spectral density (PSD) of the noise. This proposed method is derived from the theory of the traditional Allan variance method (TAVM) and a priori information of the mixed noise. The simulation result of the band-pass mixed noise with the IAVM is compared with the autoregressive (AR) model. And the results show that the IAVM could distinguish the white noise and the $1 / f$ noise in the band-pass mixed noise clearly.
\end{abstract}

\section{Introduction}

TAVM provides a way of characterizing measurement processes in the time domain and it also has an internal relationship with the PSD of the signal. TAVM can also be used to provide a measure of the behavior of the variability of a quantity as it is averaged over different sampling time periods, which results in a better convergence and the possibility to distinguish the types of noise directly. In general, the TAVM is used to distinguish the components of the system noises and analyze the intensities of the different types of noise in zener-diode [1], digital and analog DC voltmeters [2] and magnetic sensors [3], and it is also used to evaluate the system performance of the instruments such as DC nanvoltmeters [4], GNSS_INS systems [5] and inertial sensor [6, 7]. But the TAVM hasn't been used to analyze the noise properties and the performance of the direct-sampling digital radiometer [8] and the direct-sampling microwave radiometer [9]. The reason is that the TAVM can only be used for the low-pass noise characteristics analysis, while the noise included in the measurements of the direct-sampling radiometers are band-pass noise. To the band-pass noises, the TAVM cannot obtain the correct results, and very few literatures have investigated this problem.

So, this paper has proposed an IAVM to solve the above problem. This IAVM is based on the theory of the TAVM and a priori information of the band-pass mixed noise and finally it is studied and deduced through numerical simulation. This IAVM can be used to analyze the noise properties of the front end of the direct digitization receivers and then guide the procedure of design and implementation of them [10,11]. Based on the band-pass mixed noise generation model which is established through the SIMULINK in MATLAB [12], this paper utilizes this IAVM and the TAVM to analyze the characteristics of the band-pass mixed noise which consists of the band-pass Gaussian white noise and the band-pass 1/f noise, these noises are the main noise in the microwave radiometers [13, 14]. Meanwhile, the AR model $[15,16]$ has been used to calculate the PSD of the single band-pass Gaussian white noise and the single band-pass 1/f noise. The results show that this IAVM get the similar results with the AR model, which confirms the validity of the IAVM in the application of the band-pass mixed noise characteristics analysis. In addition, compared with the AR model the IAVM can easily operate and distinguish the weak noises from the system noise. 


\section{The Improved Allan Variance Method}

To an infinite time series of the quantity $y(t)$, if we divide it into many adjacent segments of duration $\tau$ and $\bar{y}_{k}$ represents the average value of $y(t)$ in the $k$ th interval, then the TAVM is defined as [4]:

$$
\sigma^{2}=\frac{1}{2}\left\langle\left(\bar{y}_{k+1}-\bar{y}_{k}\right)^{2}\right\rangle
$$

where the angular bracket denotes an infinite time average. In practice, a finite number $\mathrm{N}$ of the measurements is carried out with a minimum interval $\tau_{0}$. If the adjacent intervals $\tau_{0}, 2 \tau_{0}, 3 \tau_{0}, \ldots$, are constructed and then the traditional Allan variance of it can be estimated from:

$$
\sigma^{2}(\tau)=\frac{\sum_{k=1}^{M}\left[\bar{y}_{k+1}(\tau)-\bar{y}_{k}(\tau)\right]^{2}}{2 M}
$$

where $\mathrm{M}$ is the number of pairs, $[N / n]-1$, of $\bar{y} \cdot[N / n]$ denotes the number of the adjacent segments of the sample sequence, the bracket denotes the round down operation, $\mathrm{n}$ represents the number of the samples in each interval, and $\tau=n \tau_{0}$ denotes the lasting time of each interval.

The reason why the TAVM can be used to distinguish the types of noise directly is that it has an internal relation with the PSD of the noise by the integral as below:

$$
\sigma^{2}(\tau)=2 \int_{0}^{\infty} S(f) \frac{\sin ^{4}(\pi f \tau)}{(\pi f \tau)^{2}} d f
$$

where S(f) represents the PSD of the noise which can be a single noise or a mixed noise. And this paper does focus the attention on the mixed noise characteristics analysis.

To a mixed noise consisted of an ideal Gaussian white noise and an ideal 1/f noise, the PSD of it is defined as $S(f)=h_{0} f^{0}+h_{-1} f^{-1}$, where $h_{0}$ and $h_{-1}$ are the intensity coefficients for the Gaussian white noise and the 1/f noise, respectively. Based on the equation (3), the traditional Allan variance of the mixed noise is :

$$
\sigma_{m}^{2}(\tau)=h_{0} / 2 \tau+2 h_{-1} \ln 2
$$

where the subscript $m$ represents the mixed noise and the first item in the right side of the equation (4) represents the relationship between the traditional Allan variance and the PSD for the ideal Gaussian white noise, and the second item in the right side represents the relationship between the traditional Allan variance and the PSD for the ideal $1 / f$ noise.

It is worth noting that the result in the equation (4) based on the precondition that the PSD of the noise is spread all over the frequency domain or it has an enough wide low-pass bandwidth [17]. When S(f) denotes the band-pass mixed noise which consists of Gaussian white noise and 1/f noise, then the equation (3) should be rewritten as below and the conclusion in equation (4) is no longer valid:

$$
\sigma^{2}(\tau)=2 \int_{f_{s}}^{f_{c}} S(f) \frac{\sin ^{4}(\pi f \tau)}{(\pi f \tau)^{2}} d f, \quad 0<f_{s}<f_{c}<\infty
$$

where $f_{s}, f_{c}$ denote the upper and lower frequency limits of the PSD of the band-pass mixed noise, respectively.

In order to resolve the above problem, this paper has proposed an IAVM and the definition of it is defined as below:

$$
\hat{\sigma}^{2}(\tau)=\sigma^{2}(\tau) /(1-\alpha)
$$

where $\alpha$ denotes the error modifying factor and it is different with the different types of noise. This improved method can also make the conclusion in equation (4) still valid to the band-pass mixed noise. The difference is that the $\sigma_{m}^{2}(\tau)$ in equation (4) should be rewritten as $\hat{\sigma}_{m}^{2}(\tau)$ in 
equation (7):

$$
\hat{\sigma}_{m}^{2}(\tau)=h_{0} / 2 \tau+2 h_{-1} \ln 2
$$

Based on a priori information of the mixed noise, the equation (7) can also be written as

$$
\hat{\sigma}_{m}^{2}(\tau)=\hat{\sigma}_{w}^{2}(\tau)+\hat{\sigma}_{f}^{2}(\tau)
$$

and,

$$
\begin{aligned}
& \hat{\sigma}_{w}^{2}(\tau)=\sigma_{w}^{2}(\tau) /\left(1-\alpha_{1}\right) \\
& \hat{\sigma}_{f}^{2}(\tau)=\sigma_{f}^{2}(\tau) /\left(1-\alpha_{2}\right) \\
& \sigma_{m}^{2}(\tau)=\sigma_{w}^{2}(\tau)+\sigma_{f}^{2}(\tau)
\end{aligned}
$$

where $\hat{\sigma}_{m}^{2}(\tau), \hat{\sigma}_{w}^{2}(\tau)$ and $\hat{\sigma}_{f}^{2}(\tau)$ represent the improved Allan variances of the band-pass mixed noise, band-pass Gaussian white noise and the band-pass $1 / f$ noise, respectively. $\sigma_{m}^{2}(\tau)$, $\sigma_{w}^{2}(\tau)$ and $\sigma_{f}^{2}(\tau)$ represent the traditional Allan variances of them, respectively. $\alpha_{1}$ and $\alpha_{2}$ represent the error modifying factors of the traditional Allan variance for the band-pass white noise and $1 / f$ noise, respectively. The expressions of $\alpha_{1}$ and $\alpha_{2}$ are deduced and calculated from the incomplete integral of the equation (5), and the definitions of them are defined as below:

$$
\begin{aligned}
& \alpha_{1}=\frac{2}{\pi}\left(y_{w 1}\left(x_{1}\right)+y_{w 2}\left(x_{2}\right)\right) \\
& \alpha_{2}=\frac{1}{\ln 2}\left(y_{f 1}\left(x_{1}\right)+y_{f 2}\left(x_{2}\right)\right)
\end{aligned}
$$

where $x_{1}=2 \pi f_{s} \tau, x_{2}=2 \pi f_{c} \tau$ and

$$
\begin{aligned}
& y_{w 1}\left(x_{1}\right)=\int_{0}^{x_{1}} \frac{2 \sin x-\sin 2 x}{x} d x+\frac{4 \cos x_{1}-\cos 2 x_{1}-3}{2 x_{1}} \\
& y_{w 2}\left(x_{2}\right)=\int_{x_{2}}^{\infty} \frac{2 \sin x-\sin 2 x}{x} d x-\frac{4 \cos x_{2}-\cos 2 x_{2}-3}{2 x_{2}} \\
& y_{f 1}\left(x_{1}\right)=\int_{0}^{x_{1}} \frac{\cos x-\cos 2 x}{x} d x+\left(\frac{4 \cos x_{1}-\cos 2 x_{1}-3}{4 x_{1}^{2}}+\frac{\sin 2 x_{1}-2 \sin x_{1}}{2 x_{1}}\right) \\
& y_{f 2}\left(x_{2}\right)=\int_{x_{2}}^{\infty} \frac{\cos x-\cos 2 x}{x} d x-\left(\frac{4 \cos x_{2}-\cos 2 x_{2}-3}{4 x_{2}{ }^{2}}+\frac{\sin 2 x_{2}-2 \sin x_{2}}{2 x_{2}}\right)
\end{aligned}
$$

It is clear that the error modifying factors $\alpha_{1}$ and $\alpha_{2}$ are the function of the upper and lower frequency limits $f_{c}, f_{s}$ and the sampling time $\tau$.After a further analysis we can get that $y_{w 1}^{\prime}\left(x_{1}\right) \geq 0, y_{w 2}^{\prime}\left(x_{2}\right) \leq 0, y_{f 1}^{\prime}\left(x_{1}\right) \geq 0, y_{f 2}^{\prime}\left(x_{2}\right) \leq 0$ when $x_{1}, x_{2}$ belong to the range $[0, \infty]$, and the equalities hold only when $x_{1}=2 k \pi, x_{2}=2 k \pi$ and $k=0,1,2, \cdots$, which means in the range $[0, \infty], y_{w 1}\left(x_{1}\right), y_{f 1}\left(x_{1}\right)$ are monotone increasing functions and $y_{w 2}\left(x_{2}\right), y_{f 2}\left(x_{2}\right)$ are monotone decreasing functions, which means that the sampling time $\tau$ and the modifying factors $\alpha_{1}, \alpha_{2}$ are one-to-one corresponding relations. This ensures the feasibility of the IAVM in the application of the band-pass noise characteristics analysis.

\section{The model of the band-pass mixed noise generator}

In order to validate the feasibility of the IAVM in the application of the band-pass noise characteristics analysis, this paper firstly establishes the band-pass mixed noise generation model based on the SIMULINK [18]. This mixed noise is composed of Gaussian white noise and 1/f noise and the generation model is shown in Fig. 1. Secondly, the output data of the generation model is analyzed by using the traditional and IAVM, as well as the AR model in the time domain and the frequency domain, respectively. Finally, the results of the IAVM are compared with that of the 
TAVM, and the AR model also is used to validate the feasibility of the IAVM in the application of the band-pass noise characteristics analysis. The next paragraph is a simple introduction of the main modules in the band-pass mixed noise generation model.

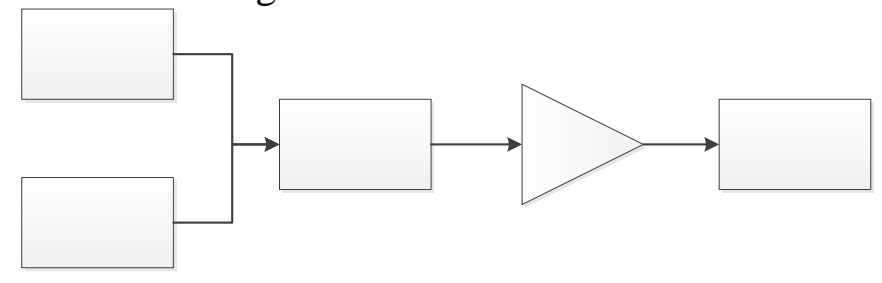

Fig. 1 The generation model of the band-pass mixed noise

In Fig. 1, the Gaussian Noise Generator is used to generate Gaussian white noise, with a mean to 0 and a variance of 0.005 . The $1 / f$ Noise Generator is used to generate $1 / f$ noise, with a mean to 0 and a variance of 0.0025 , the maximum frequency of them is $500 \mathrm{MHz}$. The pass band of the Intermediate Frequency Filter is from 10 to $110 \mathrm{MHz}$. The last module is used to transfer the output data to the workspace of MATLAB, and in each simulation process the sample data is $5.0 \times 10^{6}$.

\section{Result analysis}

In order to analyze the simulation results simply, this paper takes the following principles when use the TAVM in equation (2) and the IAVM in equation (6) to tackle the output data from the model in Fig. 1.

1. The selection of the sampling time $\tau$ follows $\tau=2^{q} \tau_{0}, q=0,1,2, \cdots$;

2. Impose the logarithm operation with base 2 to the equation (2) and (6).

In this way, the improved Allan variances of the band-pass Gaussian white noise and the band-pass $1 / f$ noise are two straight lines with a slope of $k=-1$ and $k=0$, respectively, which similar to the traditional Allan variances of the ideal Gaussian white noise and the $1 / f$ noise.

On the premise of the above processing analysis, this paper has analyzed the output data of the generation model through the TAVM and the IAVM, respectively. And the results are shown in Fig. 2 , in which the Improved $A V$ (Allan variance) represents the improved Allan variance $\hat{\sigma}_{m}^{2}(\tau)$ of the band-pass mixed noise, and the traditional $A V$ represents the traditional Allan variance $\sigma_{m}^{2}(\tau)$ of the band-pass mixed noise. It is clear that the curve of the improved Allan variance of the band-pass mixed noise is composed of two straight lines with a slope of $k=-1$ and $k=0$. According to the conclusion in equation (7), the two lines reflect the characteristics of the Gaussian white noise and the $1 / f$ noise, respectively, which in turn verify the noise components of the band-pass mixed noise. By contrast, the curve of the Traditional Allan variance of the band-pass mixed noise cannot fit the conclusion in equation (4). So, the Fig. 2 tentatively shows the feasibility of the IAVM in the application of the band-pass noise characteristics analysis.
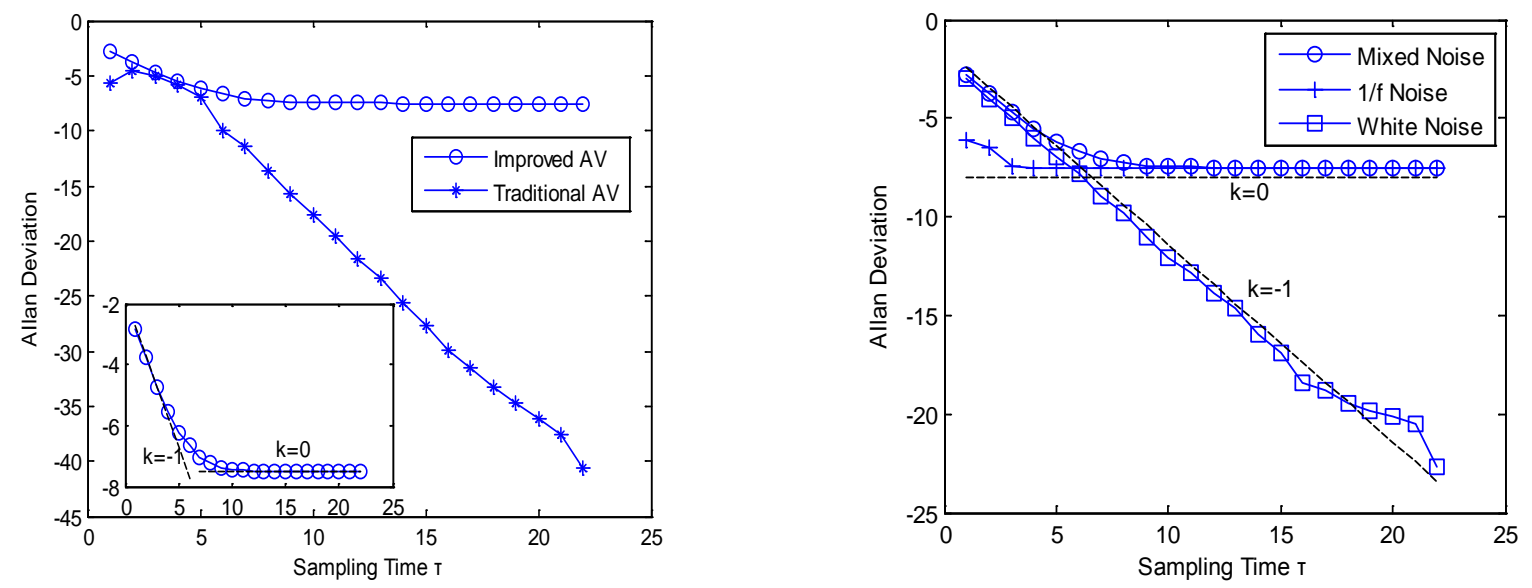
Fig. 2 The improved and traditional Allan variance (AV) of the band-pass mixed noise
Fig. 3 The improved Allan variances of band-pass noise with different types

In order to further analyze the feasibility of the IAVM in the application of the power spectrum estimation of the different types of noise in the band-pass mixed noise, this paper also calculates the improved Allan variances of the band-pass Gaussian white noise and the band-pass 1/f noise through the equation (8), and the results are shown in Fig. 3, in which we also draw the improved Allan variance of the band-pass mixed noise again. It is clear that the improved Allan variances of the band-pass Gaussian white noise and the band-pass 1/f noise are approximate to a straight line with a slope of $k=-1$ and $k=0$. This property is just like what shown in the equation (7).

According to the improved Allan variance of the band-pass $1 / f$ noise and the band-pass Gaussian white noise in Fig. 3 and the conclusion in equation (7), we can get the intensity coefficient of the $1 / f$ noise and with noise is $h_{-1}=0.0040 \mathrm{~W}$ and $h_{0}=0.4716 \times 10^{-9} \mathrm{~W}$, respectively.

In order to confirm the validity of the results referred to above of the IAVM, this paper also uses the AR model to analyze the power spectral density of the band-pass Gaussian white noise and the band-pass $1 /$ f noise, they are generated with the model in Fig. 1, separately. The results are shown in Fig. 4, in which the imaginary line represents the PSD of the band-pass Gaussian white noise and the solid line represents the PSD of the band-pass $1 / f$ noise.

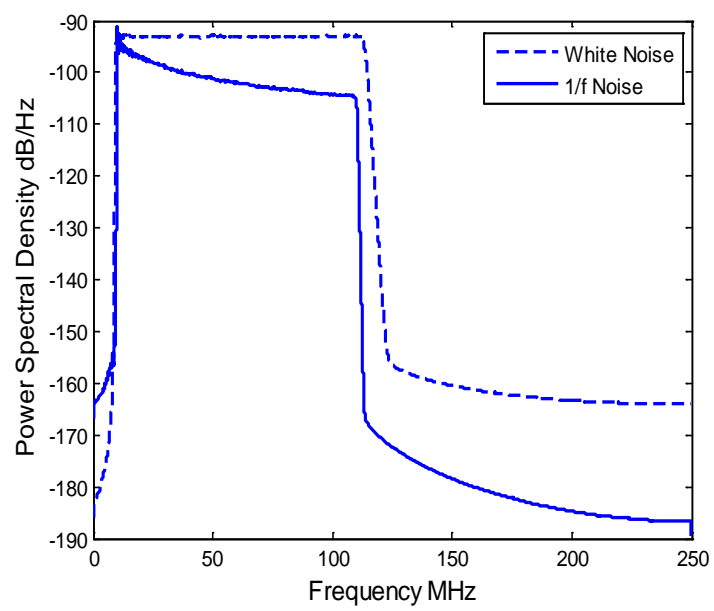

Fig. 4 The PSD of the band-pass white noise and the 1/f noise

In Fig. 4, we can get the PSD of the band-pass Gaussian white noise is $h_{0}=0.5 \times 10^{-9} \mathrm{~W} / \mathrm{Hz}$ in the pass-band range, and the intensity coefficient of the band-pass $1 / f$ noise $h_{-1}=0.0038 \mathrm{~W}$.

According to the above computation and analysis, the results show that the absolute error of the PSD of the band-pass Gaussian white noise between the IAVM and the AR model algorithm is just 5.67 percent, and the absolute error of the intensity of the band-pass $1 / f$ noise between the IAVM and the AR model algorithm is just 5.26 percent. So, the IAVM can be used better in the application of the band-pass noise characteristics analysis.

\section{Conclusion}

In this paper, we proposed an IAVM to distinguish the different types of the noise in the band-pass mixed noise accurately. The simulation was conducted to compare the IAVM with the TAVM, and the results shown that the IAVM can distinguish the white noise and the $1 / \mathrm{f}$ noise in the band-pass mixed noise clearly. At the same time, the AR model was introduced to compare the results of the noise intensities with the IAVM, and the results demonstrated that this IAVM could also accurately give out the intensities of the different types of the noise in the band-pass mixed noise.

\section{References}


[1] Witt, T.J. and D. Reymann, Using power spectra and Allan variances to characterise the noise of Zener-diode voltage standards. Science, Measurement and Technology, IEE Proceedings, 2000 147(4) 177-182.

[2] Witt, T.J., Allan variances and spectral densities for DC voltage measurements with polarity reversals. IEEE Transactions on Instrumentation \& Measurement, 20052 (54) 550-553.

[3] K. Draganová, F. Kmec, J. Blažek et al, "Noise Analysis of Magnetic Sensors Using Allan Variance,” Acta Physica Polonica A, 2014126 (1) 394-395.

[4] Witt, T.J., Using the Allan variance and power spectral density to characterize DC nanovoltmeters. IEEE Transactions on Instrumentation \& Measurement, 2001 50(2) 667 - 668.

[5] Q Zhang, X Niu, Q Chen, H Zhang, and C Shi, "Using Allan variance to evaluate the relative accuracy on different time scales of GNSS/INS systems," Measurement Science and Technology, 2013 24(8) 1659-1666.

[6] Jintao Li, and Jiancheng Fang, "Sliding Average Allan Variance for Inertial Sensor Stochastic Error Analysis,” IEEE Transactions on Instrumentation and Measurement, 2013 61(12) 3291-3300.

[7] Jintao Li, and Jiancheng Fang, "Not Fully Overlapping Allan Variance and Total Variance for Inertial Sensor Stochastic Error Analysis," IEEE Transactions on Instrumentation and Measurement, 201362 (10) 2659 - 2672.

[8] L.U. Hao, and Z.Z. Wang, Design of Twenty-four Channels Digital Correlation in Synthetic Aperture Radiometer. Remote Sensing Technology \& Application, 2011 26(3) 283-286.

[9] Zhu, L., et al. The performance analysis of direct sampling microwave radiometer. In Microwave and Millimeter Wave Technology, 2008. ICMMT 2008. International Conference on. 2008.

[10]Y Yu, Q Chang, and Y Chen, "Design and Simulation of a Fully Digitized GNSS Receiver Front-End,” Discrete Dynamics in Nature and Society, 201130 (4) 1433-1448.

[11]Yuan, Y.U., et al., Design and Validation of Direct Digitization Scheme for GNSS Receiver Front-Ends. Journal of Tianjin University, 2012.

[12]U.A. Khan, W.J. Shin, J.K. Seong, S.H. Oh, S.H. Lee, and B.W. Lee, "Feasibility analysis of the application and positioning of DC HTS FCL in a DC microgrid through modeling and simulation using Simulink and SimPowerSystem,” Physica C Superconductivity, November, 2011 (471) 1322 - 1326.

[13]P. Meinhold, R. Leonardi, et al, "Noise properties of the Planck-LFI receivers,” Journal of Instrumentation, 2009 12(4).

[14]Jeffrey R. Piepmeier, Liang Hong, Fernando A. Pellerano, “Aquarius L-Band Microwave Radiometer: 3 Years of Radiometric Performance and Systematic Effects,” IEEE Journal of Selected Topics in Applied Earth Observations and Remote Sensing, 2015 1-8.

[15]W. J. Su, H. T. Chen, "A New Algorithm for Long-Term Estimation Based on AR Model," Applied Mechanics and Materials, 2014614 440-443.

[16]Luo, X., et al., Super-resolution spectral analysis and signal reconstruction of magnetic Barkhausen noise. NDT \& E International, 2015 16-21.

[17]S. R. Stein, “The Allan variance - challenges and opportunities,” in IEEE Symposium on Frequency Control, 2009 385-839.

[18]Burrage, D.M., M.A. Goodberlet and M.L. Heron, Simulating Passive Microwave Radiometer 
Designs Using Simulink. Simulation, 2002. 78(1) 36-55. 